\title{
DEPTH FROM RELATIVE NORMAL FLOWS
}

\author{
FAN JiANG and TERRY E. WeYMOUTH* \\ Artificial Intelligence Laboratory, Electrical Engineering and Computer Science Department. University \\ of Michigan, Ann Arbor, MI 48109, U.S.A.
}

(Received 24 October 1988; in revised form 5 September 1989; received for publication 20 November 1989)

\begin{abstract}
Most of the depth from image flow algorithms has to rely on either good initial guesses. or some assumptions about the object surfaces to achieve solutions that agree with the physical world. Waxman and Sinha point out that those restrictions can be relaxed if depth is computed from a relative image flow field. Since image flow determination is relatively much more difficult than normal flow determination, it is of interest to develop an algorithm to recover depth from normal flows. In this paper, we have shown that similar results can be obtained from relative normal flow fields as from relative image flow fields. We have implemented a normal flow estimation algorithm, and applied our algorithm to recover depth from intensity images.
\end{abstract}

Depth Normal flow Relative normal flow Static stereo Dynamic stereo

\section{INTRODUCTION}

Depth recovery from image flow fields is an important but difficult problem in computer vision. A working depth recovery algorithm, as a method to determine absolute range to objects in a scene, will find a variety of applications in robots, such as collision avoidance and surface reconstruction. However, depth recovery algorithms proposed so far have not been able to provide satisfactory results. Some of the algorithms are based on solving non-linear equations, hence the solutions rely on good initial guesses; ${ }^{(1-3)}$ some of the algorithms give good solutions only on restricted occasions; ${ }^{(4-12)}$ and some of the algorithms tends to amplify effects of noise in the input data. ${ }^{(13)}$ The goal of this research is to develop an algorithm to overcome the aforementioned weaknesses.

Actually, structure from image flow fields is an illposed problem. Generally, the structure is determined from the image flow fields along with 3D motion parameters of the objects. This structure and 3D motion recovery problem is also referred to as the problem of interpreting image flow fields. It is well-known that there could be infinite number of interpretations if the object points can move independently. To obtain a unique interpretation, the one that agrees with the physical world, some constraints are needed. The most widely adopted constraint is a rigidity constraint, which assumes that the objects undergo rigid motion. Based on this constraint, equations can be derived to relate image flow vectors to the object structure and 3D motion.

*To whom correspondence should be addressed.
Interpretations can be obtained by solving these equations. As the equations are non-linear, however, there could still be many interpretations. To obtain an interpretation that agrees with the physical world, an initial guess close to the correct solution is needed. Unfortunately, it is almost impossible to find such initial guesses.

A unique interpretation can be achieved if additional assumptions are made. The assumptions should be made such that the rigid motion equations can be reduced to linear expressions or at least the number of possible solutions are sharply reduced. There are two simple assumptions each of which will make the equations linear: assuming the depth values of the object points are known, ${ }^{(12)}$ and assuming the 3D motion parameters are known. ${ }^{(14)}$ Once the equations are linear, an appropriate solution is guaranteed, and the original problem becomes rather straightforward to solve. On the other hand, the most widely used assumption that will sharply reduce the number of possible solutions is the assumption that the scene consists of planar surfaces only. ${ }^{(5.7-10.15)}$ It is shown that at most two solutions exist for a planar surface. ${ }^{(8.9)}$ In the case of dual solutions, the false solution can be eliminated by comparing the solutions with those of another planar surface of the same rigid object. ${ }^{(7.15)}$ Unfortunately, this assumption greatly limits the application of the algorithms.

Waxman and Sinha have shown that a unique interpretation can also be achieved without additional assumptions. They do this by the introduction of a reference image flow field. ${ }^{\left({ }^{13)}\right)}$ By taking the difference of the reference field and the original image flow field, a relative image flow field can be 
obtained. Then, the absolute range to the object points can be determined from this relative field. provided that the relative motion between the original camera and the reference camera is given. This formulation is attractive because it needs no additional assumptions and is hence generally applicable. A major weakness of the formulation is that the effect of noise in the input flow fields is amplified due to the differencing process in obtaining the relative flow field. The effect of noise can be suppressed to some extent, the paper suggested, by filtering the relative flow field.

In this paper, we report on a structure recovery algorithm that is an extension of Waxman and Sinha's formulation. ${ }^{(13)}$ While their algorithm recovers depth from optical flow fields, we suggest that similar results can be obtained from normal flow fields, which are easier to obtain from image intensity data (Section 2). We have implemented a normal flow estimation algorithm, and applied our algorithm to intensity images to recover depth. Results from experimental runs of that algorithm are shown in Section 3, and in Section 4, a conclusion is drawn.

\section{DEPTH FROM RELATIVE NORMAL FLOW FIELDS}

As mentioned earlier, our depth recovery algorithm is an extension to Waxman and Sinha's algorithm. ${ }^{(13)}$ While their algorithm determines depth from a relative field of optical flows, ours determines depth from a relative field of normal flows. A normal flow vector is the component of the corresponding optical flow vector in the normal direction of the isobrightness contours. A normal flow field can be estimated directly from a sequence of intensity images based on a constant-brightness constraint, while the computation of an optical flow field needs additional constraints such as the heuristic smoothness assumption. ${ }^{(16)}$ In many cases, the additional constraints lead to an erroneous optical flow field. ${ }^{(8)}$

In the following, we first introduce Waxman and Sinha's formulation; ${ }^{(13)}$ then we show that similar formulation exists for the depth recovery from normal flow fields; and finally, we show that the concept of relative normal flows can also be used to determine depth from stereo images.

\subsection{Waxman and Sinha's formulation}

In the formulation, a dual camera imaging system is adopted. It is assumed that the cameras can be modeled by pin-hole cameras. One such imaging system is shown in Fig. 1, along with the coordinate systems. As shown in the figure, the image coordinates are denoted by small letters $(x, y)$, and the world coordinates by capital letters $(X, Y, Z)$.

For each camera, the optical flow field corresponds to the time-varying projection of object surface texture, due to the relative rigid body motions between objects in the scene and the camera. The equations

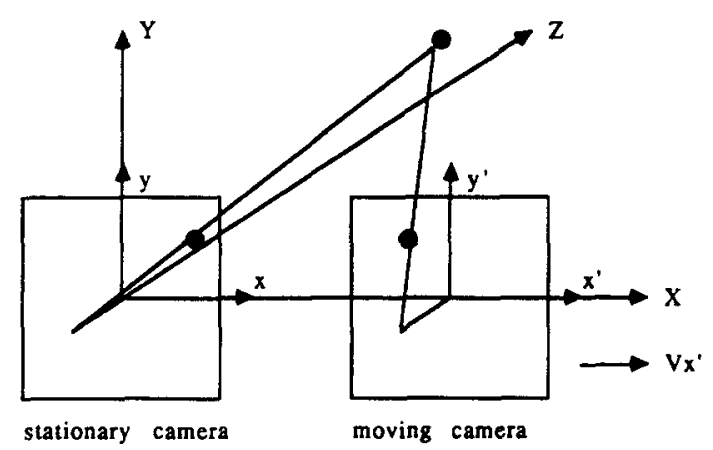

Fig. 1. Imaging system.

relating optical flow vectors to relative 3D motion and depth to points in the scene can be derived, and are given as follows

$$
\begin{aligned}
& u=-\Omega_{X} x y+\Omega_{Y}\left(1+x^{2}\right)-\Omega_{Z} y+\frac{V_{X}-x V_{Z}}{Z} \\
& v=-\Omega_{X}\left(1+y^{2}\right)+\Omega_{Y} x y+\Omega_{Z} x+\frac{V_{Y}-y V_{Z}}{Z}
\end{aligned}
$$

where $(u, v)$ is the optical flow vector at $(x, y)$, and $\left(V_{X}, V_{Y}, V_{Z}\right)$ is the vector of $3 \mathrm{D}$ translation parameters, and $\left(\Omega_{X}, \Omega_{Y}, \Omega_{Z}\right)$ is the vector of $3 \mathrm{D}$ instantaneous rotational parameters between the camera and the object.

It is obvious that recovering depth values from single optical flow field will end up with solving nonlinear equations, as the 3D motion parameters are generally unknown. To reach an algorithm that determines depth based on linear equations, the formulation introduces a reference flow field. In the formulation, a dual camera imaging system is adopted. The reference flow field is provided by the second camera. It is assumed that the relative motion between the two cameras is known, and that the two cameras come so close to each other at the time of consideration that correspondence between the two image planes is easy to establish. A relative optical flow field can then be computed by taking the difference of the two flow fields. If $(\Delta u, \Delta v$ is used to denote the relative flow vectors, $\left(\Delta V_{X}, \Delta V_{Y}, \Delta V_{Z}, \Delta \Omega_{X}, \Delta \Omega_{Y}, \Delta \Omega_{Z}\right)$ the relative motion parameters between the two cameras, then

$$
\begin{aligned}
\Delta u=- & \Delta \Omega_{X} x y+\Delta \Omega_{Y}\left(1+x^{2}\right)-\Delta \Omega_{Z} y \\
& +\frac{\Delta V_{X}-x \Delta V_{Z}}{Z} \\
\Delta v= & -\Delta \Omega_{X}\left(1+y^{2}\right)+\Delta \Omega_{Y} x y+\Delta \Omega_{Z} x \\
& +\frac{\Delta V_{Y}-y \Delta V_{Z}}{Z} .
\end{aligned}
$$

As can be seen from equation (2), depth values to the object points can be determined from the relative optical flows and the camera relative motion. The computation is rather straightforward. 


\subsection{Depth from relative normal flow field}

Our formulation adopts the same dual camera imaging system as Waxman and Sinha's, ${ }^{(13)}$ and makes the same assumptions about relative position and relative motion between the two cameras. The major difference is that we use normal flow fields while they used optical flow fields. Other differences include: in our formulation, depth can be recovered as long as the relative motion between the two cameras is not purely rotational, and the computation is simple and straightforward; while in their formulation, depth cannot be recovered if there is no relative translation in depth direction, and the computation also relies on the determination of the focus of expansion of the relative flow field.

A normal flow vector is the component of the corresponding optical flow vector in the normal direction of the iso-brightness contours at the image point. If the normal direction of an image point $(x, y)$ makes an angle $\alpha$ with the $x$-axis, an equation relating the normal flow vector of the point to the $3 \mathrm{D}$ motion and the depth to the point can be derived

$V_{n}=u \cos \alpha+v \sin \alpha$

$$
\begin{aligned}
= & \left(-\Omega_{X} x y+\Omega_{Y}\left(1+x^{2}\right)-\Omega_{Z} y+\frac{V_{X}-x V_{Z}}{Z}\right) \\
& \times \cos \alpha+\left(-\Omega_{X}\left(1+y^{2}\right)+\Omega_{Y} x y+\Omega_{Z} x\right. \\
& \left.+\frac{V_{Y}-y V_{Z}}{Z}\right) \sin \alpha
\end{aligned}
$$

where $V_{n}$ denotes the magnitude of the normal flow vectors.

Similarly, a relative normal flow field can be obtained by taking the difference of the two normal flow fields of the cameras. And an equation relating the relative normal flow vectors to the relative $3 \mathrm{D}$ motion and the depth to the points is given as follows

$$
\begin{aligned}
\Delta V_{n}= & u \cos \alpha+v \sin \alpha \\
= & \left(-\Delta \Omega_{X} x y+\Delta \Omega_{Y}\left(1+x^{2}\right)-\Delta \Omega_{Z} y\right. \\
& \left.+\frac{\Delta V_{X}-x \Delta V_{Z}}{Z}\right) \cos \alpha+\left(-\Delta \Omega_{X}\left(1+y^{2}\right)\right. \\
& \left.+\Delta \Omega_{Y} x y+\Delta \Omega_{Z} x+\frac{\Delta V_{Y}-y \Delta V_{Z}}{Z}\right) \sin \alpha
\end{aligned}
$$

where $\Delta V_{n}$ denotes the magnitude of the relative normal flow vectors.

In equation (4), the variables are either known or can be estimated from the images except the depth $Z$. If the relative motion between the cameras is not purely rotational, the $Z$ values can then be locally and uniquely determined. Thus we see that depth can also be determined from relative normal flow fields. As the equation contains no motion par- ameters associated with the object, the equation can be used in the cases of multiple objects, and the objects can have independent general motion.

Equation (4) becomes extremely simple if the relative motion parameters are zeros except one translational component. Let's assume that only $\Delta V_{X}$ is non-zero, the equation is reduced to

$$
\Delta V_{n}=\frac{\Delta V_{X}}{Z} \cos \alpha \text {. }
$$

Hence

$$
Z=\frac{\Delta V_{X}}{\Delta V_{n}} \cos \alpha .
$$

Similar results can be obtained for the cases of only $\Delta V_{Y}$ or $\Delta V_{Z}$ are non-zeros.

\subsection{Relative normal flow and close baseline stereo images}

The concept of relative normal flow can also be applied to determine depth from stereo images. In reference (13) the images of the dual camera system are referred to as dynamic stereo images. In the following, we shall show that static stereo images are a special kind of dynamic stereo image. A requirement is that the baseline between the two cameras should be close, so that the constant-brightness constraint is valid to compute normal flows from the images.

Consider a scene containing stationary objects only. In the dynamic stereo case, the stationary camera will generate a normal flow field with zero values everywhere. The relative flow field is determined solely by the normal flow field of the moving camera. Now let us assume that the moving camera takes the same positions in space as the static stereo cameras when it produces images. As shown in Fig. $2, P_{1}, P_{2}$ and $P_{3}$ indicate the locations of the static stereo cameras, as well as the positions occupied by the moving camera when it generates images. We adopt a three-camera static stereo imaging system, because we estimate normal flow fields from sequences of three images. In the figure, $I_{1}, I_{2}$ and $I_{3}$ indicate the images generated by the moving camera at time instants $t_{1}, t_{2}$ and $t_{3}$, and $I_{1}^{\prime}, I_{2}^{\prime}$ and $I_{3}^{\prime}$ indicate the images generated by the stereo cameras at the same time instant $t$. As the objects are station-

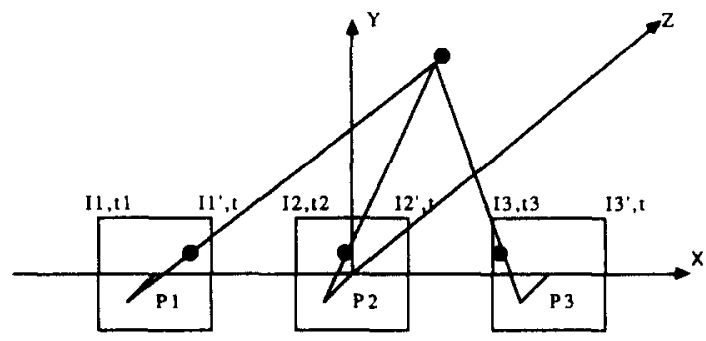

Fig. 2. Images from moving camera and stereo cameras. 
ary, it follows that

$$
I_{1}=I_{1}^{\prime} \quad I_{2}=I_{2}^{\prime} \quad I_{3}=I_{3}^{\prime}
$$

which means that the image sequence given by the moving camera is the same as the one given by the static stereo cameras.

Hence, static stereo images can be treated as a special kind of dynamic stereo image. A relative normal flow field can be estimated from the images, and depth of the object points can be determined. Obviously, the $3 \mathrm{D}$ relative motion parameters in equation (4) are determined by the relative positions and orientations of the static stereo cameras.

\section{EXPERIMENT AND RESULTS}

In this section, we present some experiments to illustrate the computation of depth from relative normal flows. Actually, the experiments will not be interested if the normal flows are given as input. It will be interesting if depth is computed from intensity images. To do so, we need to implement a normal flow estimation algorithm. In this section, we will first present our implementation of normal flow estimation, then show the experiment results of depth recovery from both dynamic stereo images and static stereo images based on our depth from relative normal flow algorithm.

\subsection{Normal flow estimation}

The constraint and the equation for the normal flow estimation is given in reference (4). The major part of the estimation is to estimate the intensity gradients $I_{x}, I_{y}$ and $I_{t}$. Our implementation includes two steps: smoothing of image intensities and estimation of intensity gradients.

Image smoothing is done by convolving the images with a two-dimensional Gaussian filter. The size of the filter is governed by the standard deviation of the Gaussian function. The purpose of smoothing is to reduce the effect of noise in intensity gradient estimation. It is well-known that there are many sources of noise in the imaging process, such as lens distortion and image digitization. A good way to reduce the noise effect is to smooth the images. In image processing, Gaussian filters are widely used for smoothing purposes. In our experiments, the standard deviations of the Gaussian filters are handpicked, and big values should be used if the image motion is large.

In this implementation, we borrow Horn and Schunck's idea ${ }^{(16)}$ to estimate intensity gradients. Their algorithm uses two images to estimate intensity gradients, while ours uses three images, i.e.

$$
\begin{aligned}
I_{x}= & \frac{1}{6}\left(I_{i, j+1, k-1}-I_{i, j, k-1}+I_{i+1, j+1, k-1}\right. \\
& -I_{i+1, j, k-1}+I_{i, j+1, k}-I_{i, j, k} \\
& +I_{i+1, j+1, k}-I_{i+1, j, k}+I_{i, j+1, k+1} \\
& \left.-I_{i, j, k+1}+I_{i+1, j+1, k+1}-I_{i+1, j, k+1}\right)
\end{aligned}
$$

$$
\begin{aligned}
I_{y}= & \frac{1}{6}\left(I_{i+1, j+1, k-1}-I_{i, j+1 . k-1}+I_{i+1 . j . k-1}\right. \\
& -I_{i, j, k-1}+I_{i+1, j+1 . k}-I_{i, j+1 . k} \\
& +I_{i+1 . j, k}-I_{i, j, k}+I_{i+1 . j+1 . k+1}-I_{i, j+1 . k+1} \\
& +I_{i+1 . j, k+1}-I_{i, j, k+1} \\
I_{t}= & \frac{1}{8}\left(I_{i+1 . j+1, k+1}-I_{i+1 . j+1, k-1}+I_{i+1, j, k+1}\right. \\
& -I_{i+1 . j, k-1}+I_{i, j+1, k+1} \\
& \left.-I_{i, j+1, k-1}-I_{i, j, k+1}-I_{i, j, k-1}\right) .
\end{aligned}
$$

From our experience, three images tend to give more consistent results than two images.

\subsection{Dynamic stereo images}

The setup of two cameras in known relative motion provides dynamic stereo. ${ }^{(13)}$ Section 2.3 has presented two equations for the depth recovery from dynamic stereo images, equation (4) for the case of general relative motion, and equation (6) for a restricted case of translational relative motion. While both equations are good for the scene of multiple objects undergoing independent motion, we used equation (6) because fewer variables introduce less error.

Four experiments were conducted to show the performance of the algorithm with dynamic stereo images. In each of the experiments, five images $I_{1} \ldots I_{5}$ are given as a dynamic sequence. As shown in Fig. $3, I_{1}, I_{3}, I_{5}$ form the moving camera sequence and $I_{2}, I_{3}, I_{4}$ form the stationary camera sequence. The two sequences share image $I_{3}$, as the imaging system is designed such that the two cameras take the second image at the same time and same position. For each sequence, a normal flow field is computed, and the values are assigned to the image points of the second image, which is image $l_{3}$ for both sequences. So, the registration problem of the two normal velocity fields is automatically solved. The relative normal flow field is obtained by taking the difference of the two normal flow fields.

In these experiments, the images were design synthetically, one for a stationary camera, and the other for a camera translating along $\boldsymbol{x}$-axis. Same synthetic camera models and same object models were used in these experiments. Both the stationary camera model and the moving camera model have the focal lengths of 100 pixels. The translational velocity of the moving camera simulated by separation of the

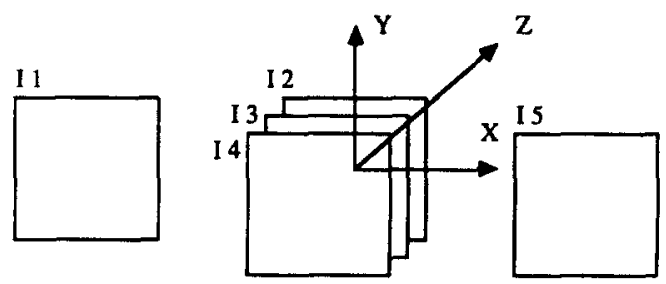

Fig. 3. Dynamic stereo sequence. 


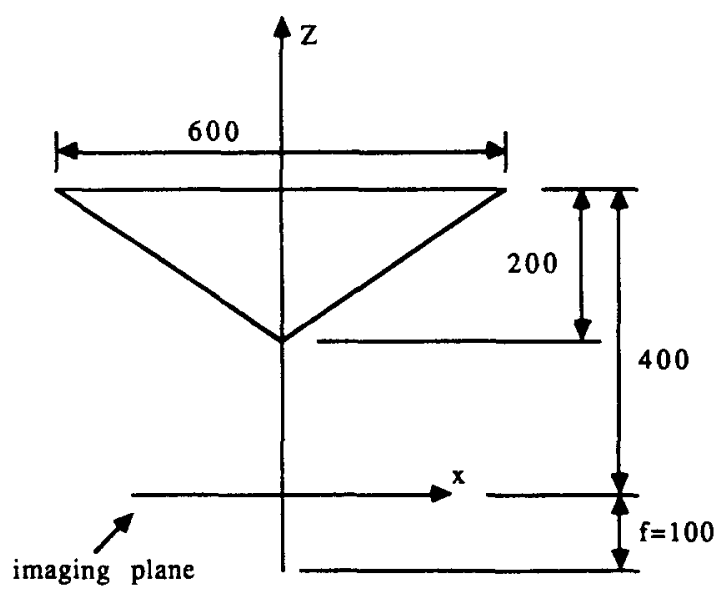

Fig. 4. Pyramid and camera. three cameras is set to be 2 pixels per unit time. A pyramid is designed to be the object being viewed. As shown in Fig. 4, the pyramid has the base of 600 pixels by 600 pixels, and the height of 200 pixels. The pyramid is placed so that its base is 400 pixels in front of the imaging planes of the three cameras. The image points in each visible side of the pyramid are assigned to have the same intensity values. The four sides have the intensity values of $50,100,150$ and 200 . The background has the intensity value of 255.

The experiments differed in the motion of the objects. For the first three experiments, the pyramid is set to rotate about $Z$-axis at different angular velocities. The purpose of these experiments is to illustrate the effect of rotation on the accuracy of normal velocity estimation. Table 1 shows the angu-

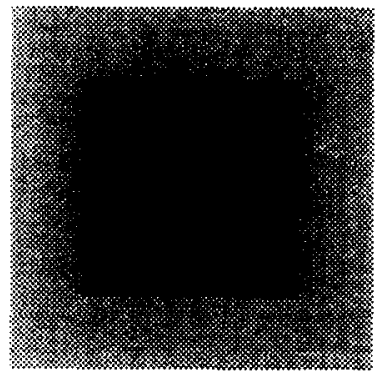

I3

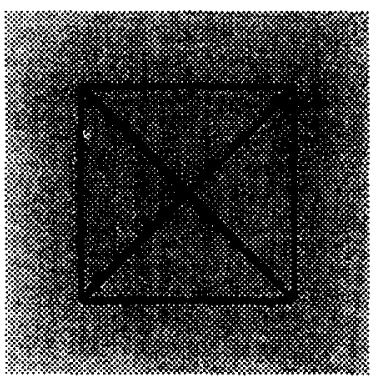

dps

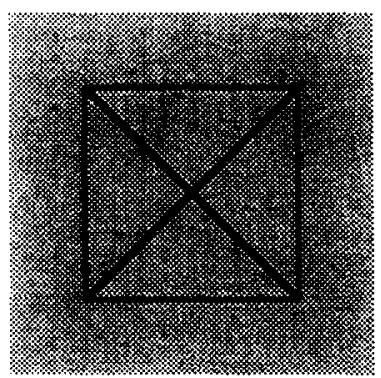

dpm

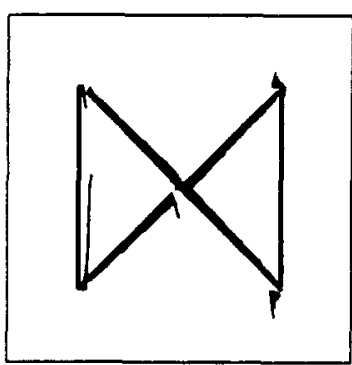

$V_{\text {ror }}$

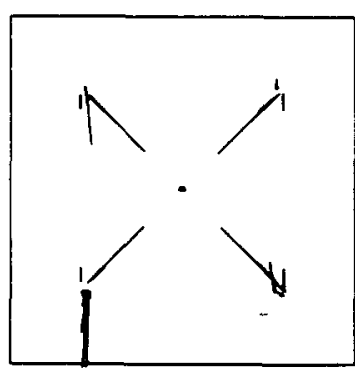

$V_{\text {ns }}$

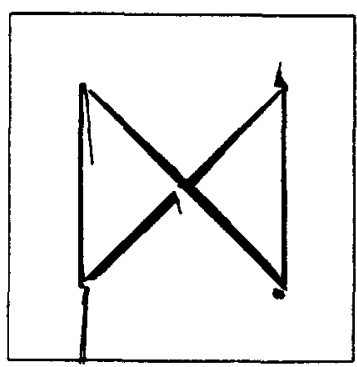

$V_{n m}$

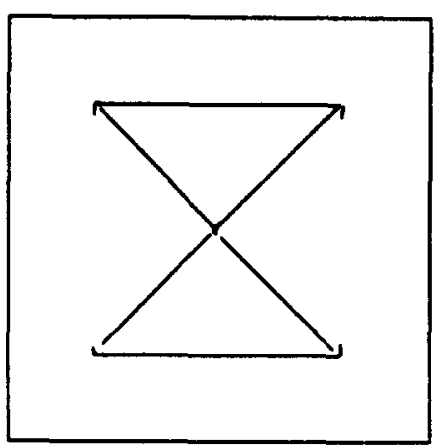

(c)

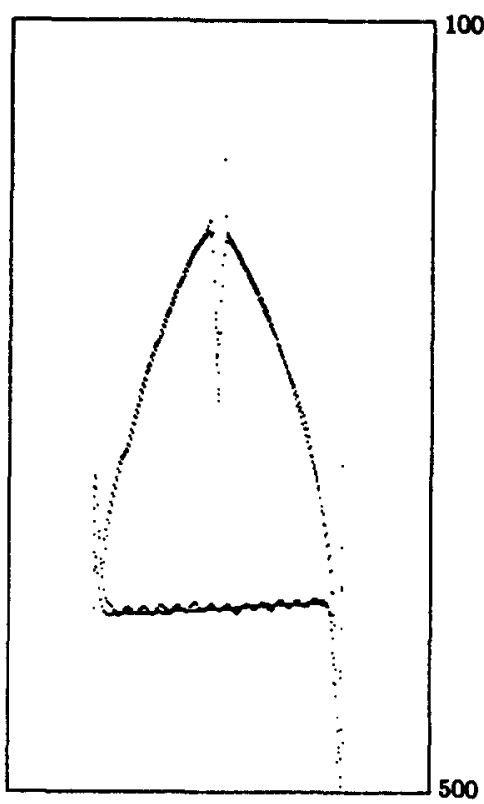

(d)

(a)

(b)

Fig. 5. Pyramid: $V=0, \Omega=\Omega_{z}=0.1$. 
Table 1.

\begin{tabular}{ccc}
\hline Experiment & $\begin{array}{c}\text { Angular velocity } \\
\left(\Omega_{z}\right)\end{array}$ & $\begin{array}{c}\text { Maximum relative error } \\
\left(\delta V_{n} / V_{n}\right)\end{array}$ \\
\hline 1 & 0.1 & 0.01 \\
2 & 0.5 & 0.02 \\
3 & 1.0 & 0.12 \\
\hline
\end{tabular}

lar velocities of the pyramid and the maximum relative errors of the estimated relative normal flow fields.

The angular velocities in the table are of the units of degrees per unit time. It can be seen from the table that when the angular velocity gets bigger, so does the maximum relative error.
Figure 5 shows the figures associated with experiment 1 . For display purposes, the intensity values of the background in the gray level images is changed from 255 to 230. In Fig. 5(a), I3 shows the image $I_{3}$ of the dynamic sequence. Figure 5(a) dps shows the difference image of the images $l_{2} . I_{4}$ of the stationary camera, Fig. 5(a) dpm shows the difference image of the images $I_{1}, I_{5}$ of the moving camera. In Fig. 5 (b), $V_{n r}$ shows the relative normal flow field, $V_{n s}$ the normal flow field of the stationary camera, $V_{n m}$ the normal flow field of the moving camera. We only estimate the normal flow values at zero crossing edge points, because the estimates are more reliable at the positions where the intensity gradient values are high. ${ }^{(5)}$ These fields show the normal flow values at those points. Note that we have also disregarded the

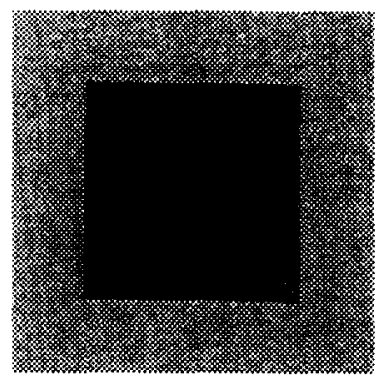

13

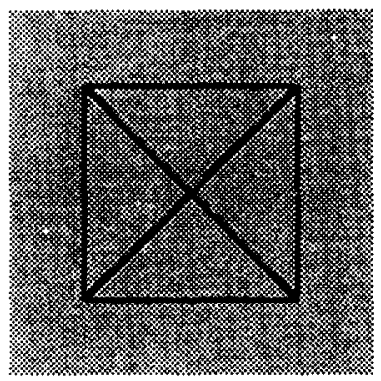

dps

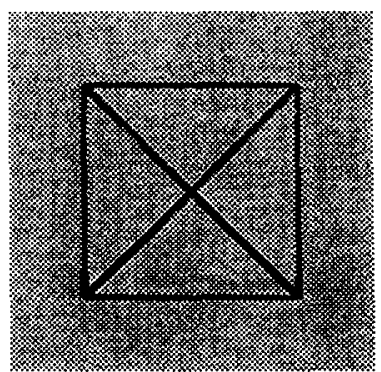

$\mathrm{dpm}$

(a)

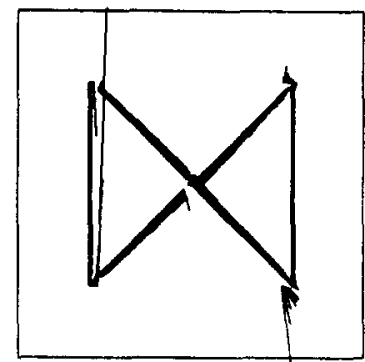

$V_{n r}$

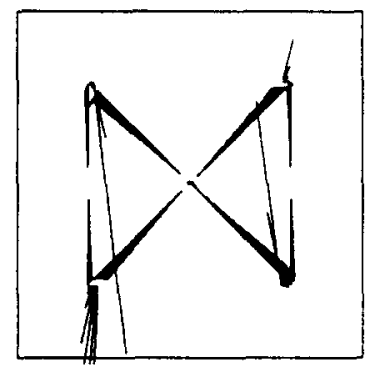

$V_{n s}$

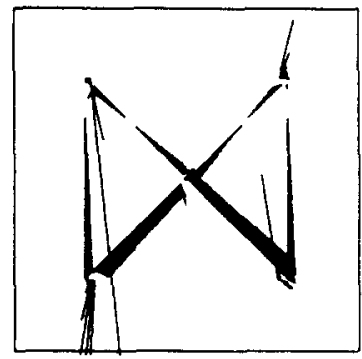

$V_{n m}$

(b)

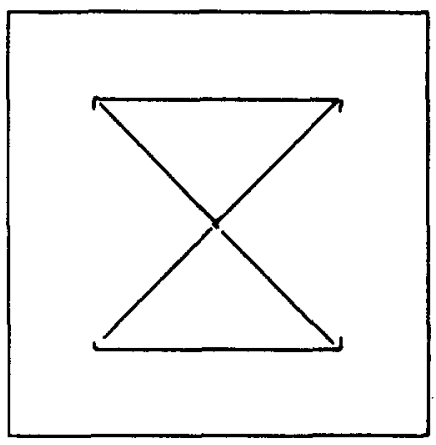

(c)

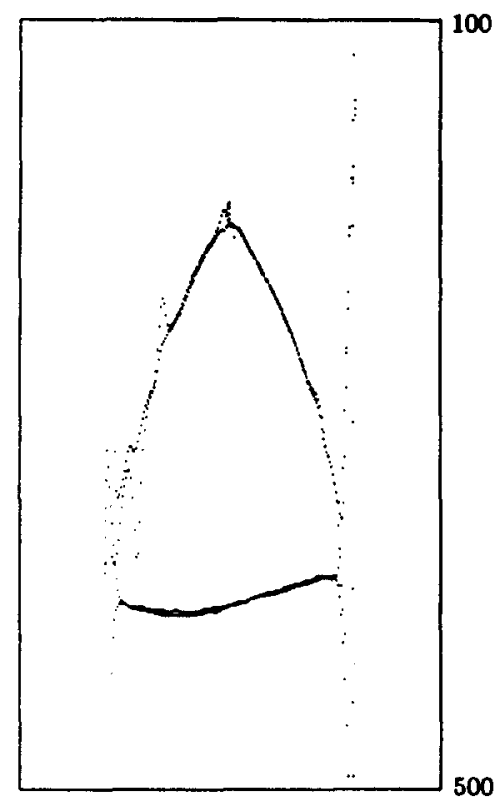

(d)

Fig. 6. Pyramid: $V=0, \Omega=\Omega_{z}=0.5$. 


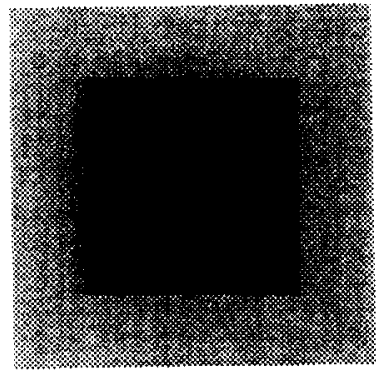

I3

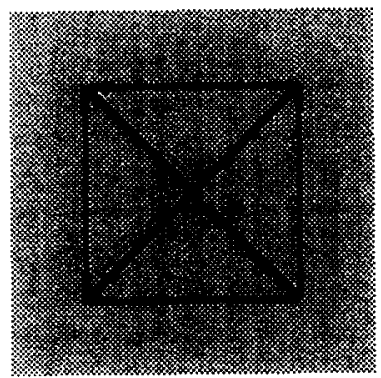

dps

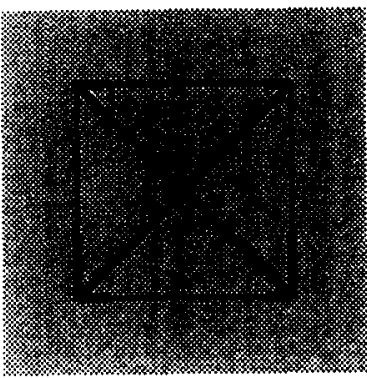

dpm

(8)

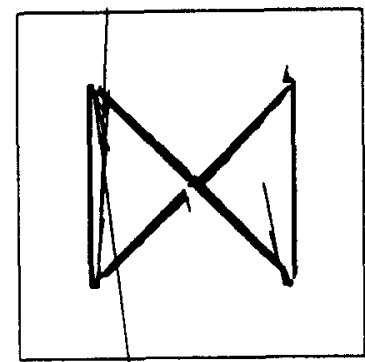

$V_{n r}$

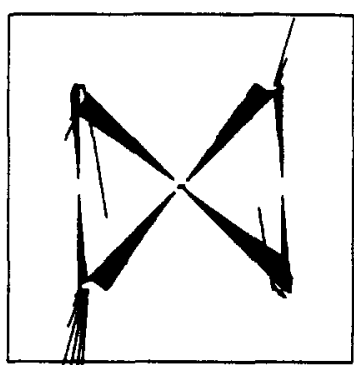

$V_{\text {no }}$

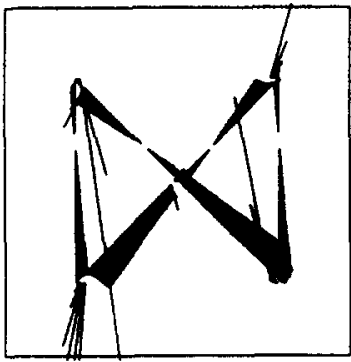

$V_{n m}$

(b)

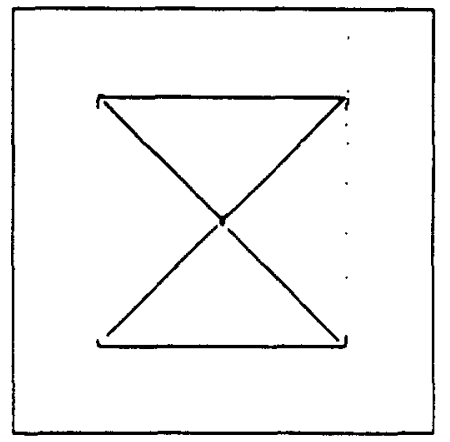

(c)

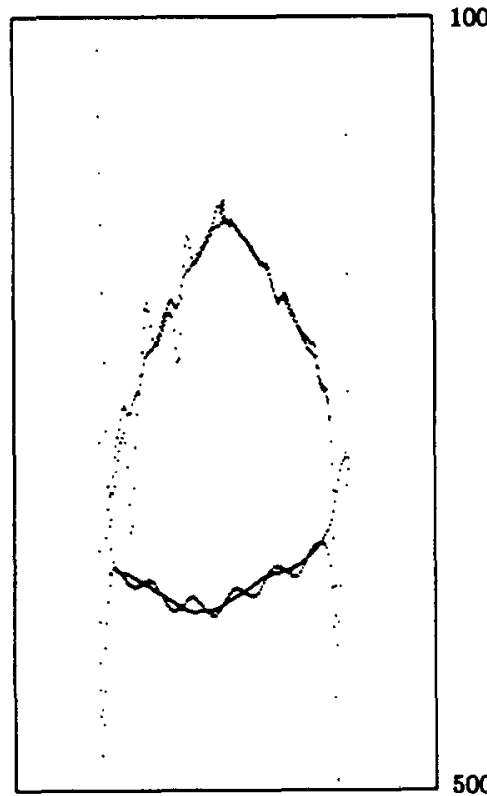

(d)

Fig. 7. Pyramid: $V=0, \Omega=\Omega_{Z}=1.0$.

zero crossing edge points whose $\alpha$ values are zeros, as the depth values of these points cannot be determined from their relative normal flow (see equation (6)). Figure 5(c) shows edge points of image $I_{3}$ whose depth values can be determined. Figure 5(d) shows the projection of the recovered depth values onto the $Y-Z$ plane of the world coordinate system.

From Fig. 5(d), it can be seen that the errors of the depth values for cornerpoints are very big. This is due to the fact that the intensity gradients of the corner points is not well-defined. In the following analysis, we simply disregard these points. After disregarding these points, it can be seen that the maximum relative error comes from the points on the base of the pyramid. This is where the motion induced by the rotation is at its maximum value. It can also be seen that some strong edge points, the points on the other two base lines of the pyramid, are not shown. This is due to the fact that the relative normal flow have zero values at these points, because the translational velocity of the moving camera has no $y$ component, while the normal vectors of these edge points are in $y$ direction. A possible way to solve this problem is to add another set of cameras to simulate a translation in $y$.

Figures 6 and 7 show the corresponding figures associated with experiments 2 and 3 .

In the fourth experiment, the pyramid is designed to rotate about the axis $(1,1,1)$ with the angular velocity of 0.5 degrees per unit time, and translate 


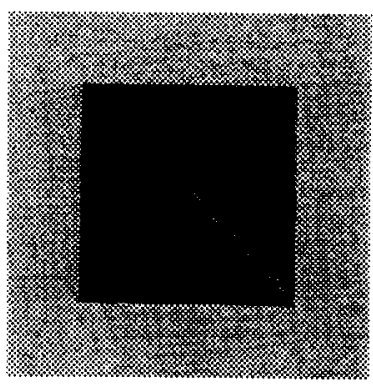

13

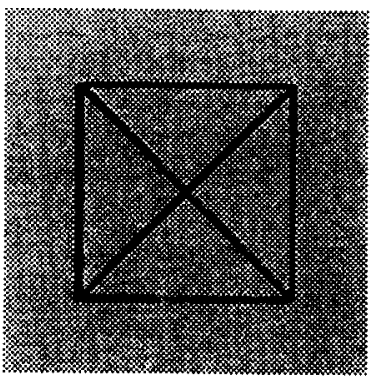

dps

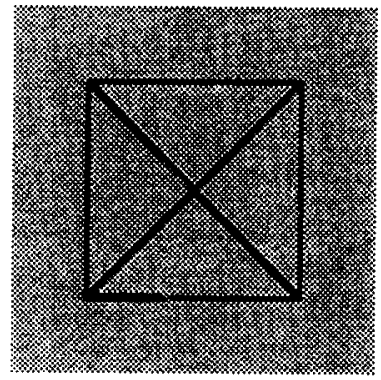

dpm

(a)

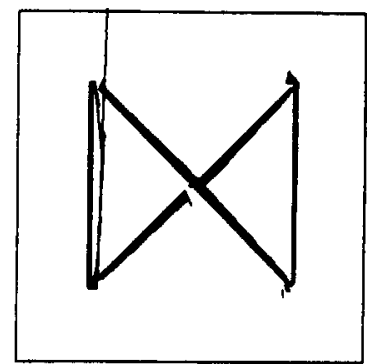

$V_{\text {nr }}$

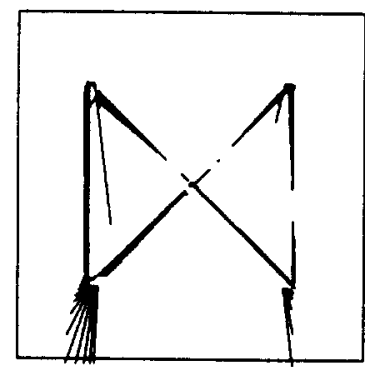

$V_{\text {ns }}$

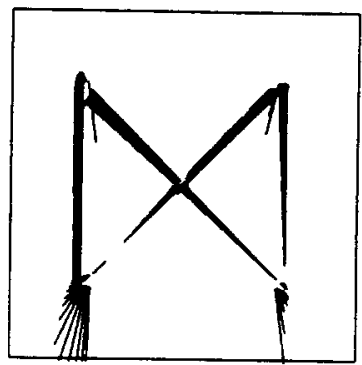

$V_{n m}$

(b)

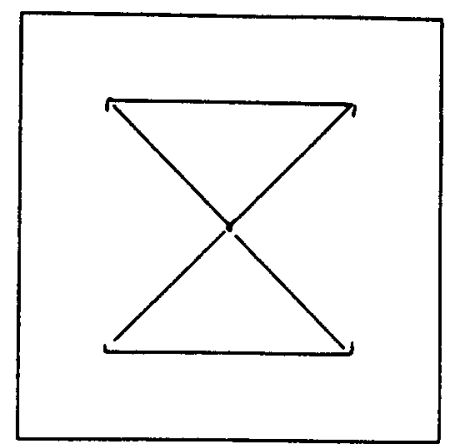

(c)

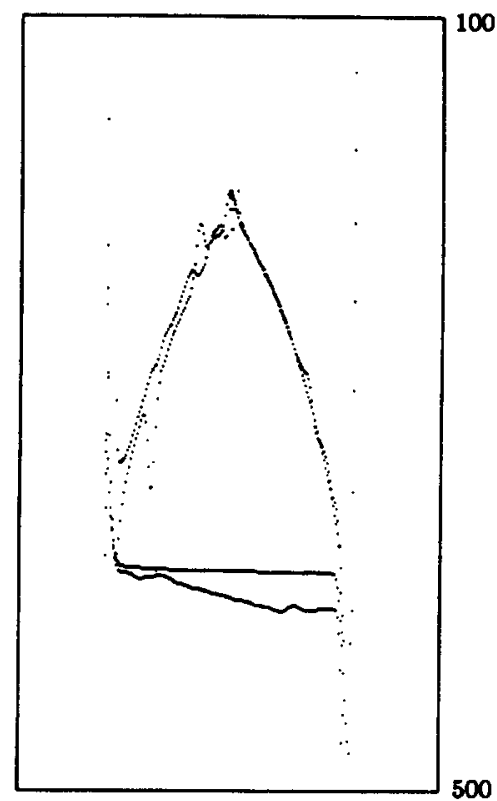

(d)

Fig. 8. Pyramid: 3D motion.

with the velocity of $(0.5,1,1.5)$ pixels per unit time. The purpose of this experiment is to illustrate the performance of the algorithm with the dynamic stereo images when the object is in general motion. Figure 8 shows the figures associated with this experiment. As can be seen from Fig. 8(d), the maximum relative error comes from the points on the base of the pyramid. This is also where the rotation induced motion is maximum. The maximum relative error of the relative normal flow is 0.05 , or $5 \%$.

As the accuracy of the depth values depends solely on the accuracy of the relative normal flows, these experiments actually show that our implementation of the normal flow estimation provides practically good results when applied to dynamic stereo images.

\subsection{Static stereo images}

As explained in Section 2.3, the concept of relative normal flows can be used to determine depth from static stereo images. Again, the key is to estimate normal flows from images. Here, we present three experiments that recover depth from static stereo images based on normal flows. As before, three images from each sequence are used to compute the relative normal flow field.

3.3.1. Synthetic pyramid. This experiment illustrates the performance of our algorithm with controlled data. In the experiment, the cameras and the pyramid are the same as in the dynamic stereo case, 


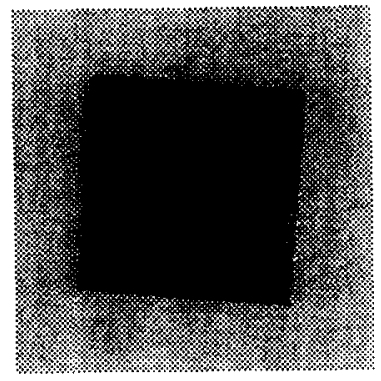

I2



dp

(a)

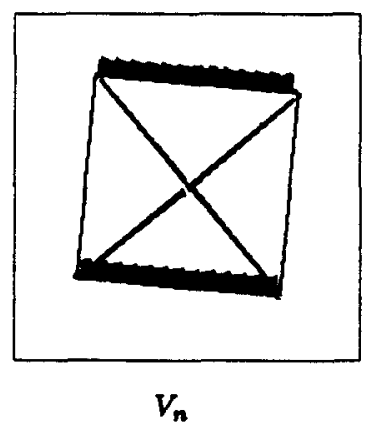

(b)

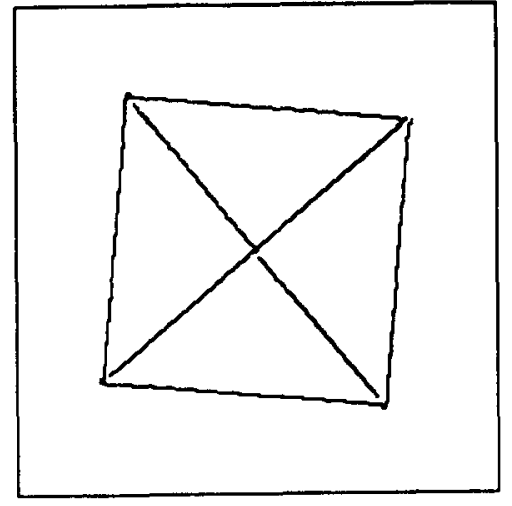

(c)

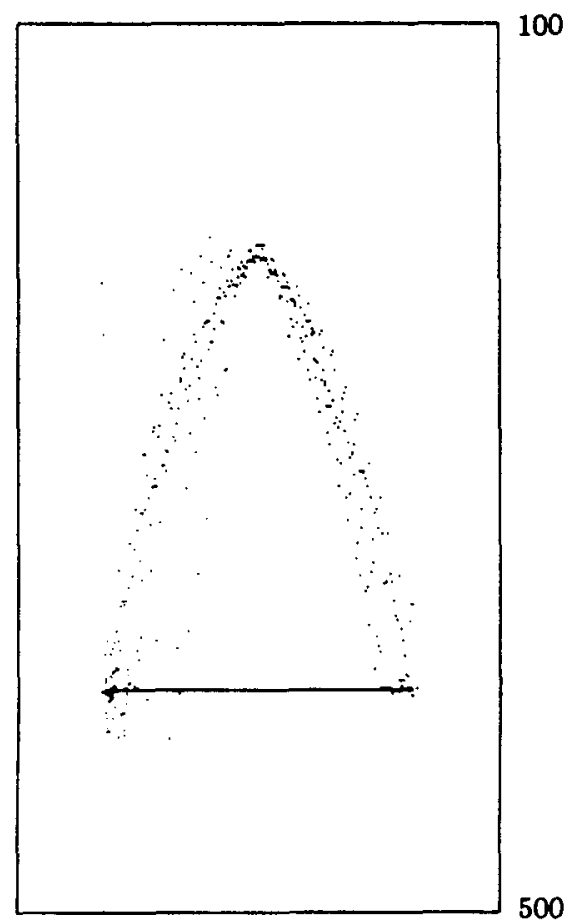

(d)

Fig. 9. Synthetic pyramid.

only that the pyramid is stationary this time to generate static stereo images. The two cameras have laterally a distance of 2 pixels between them.

Figure 9 shows the figures associated with this experiment. In Fig. 9(a), I2 shows the second image of the sequence, dp shows difference image of the images $I_{1}, I_{3}$ of the sequence. Again, for displaying purposes, the background intensity values in $I_{2}$ are changed from 255 to 230 , and the difference image is subtracted from an image with uniform intensity values of 230 . Figure 9 (b) shows the relative normal flow values of strong edge points. Figure $9(\mathrm{c})$ shows the strong edge points of image $I_{2}$, and Fig. $9(\mathrm{~d})$ shows the projection of the recovered depth values onto the $Y-Z$ plane of the world coordinate system. As in the case of dynamic stereo, the corner points are disregarded. The maximum relative error of the relative normal flow field is less than $1 \%$.

Compare Fig. 9(d) with Figs 5(d), 6(c), 7(d) and $8(d)$, it can be seen that 9 (d) provides better results. This is due to the following: for dynamic stereo, the objects are in general motion, the motion of the 


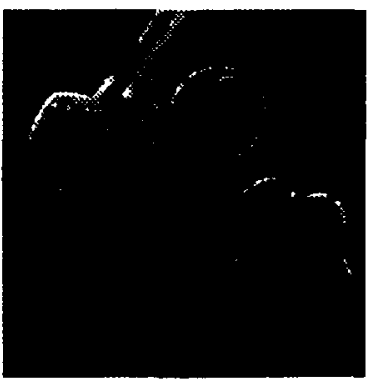

12

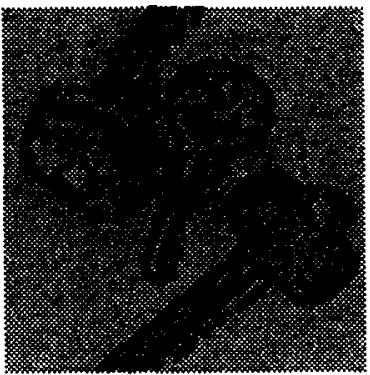

dp

(a)

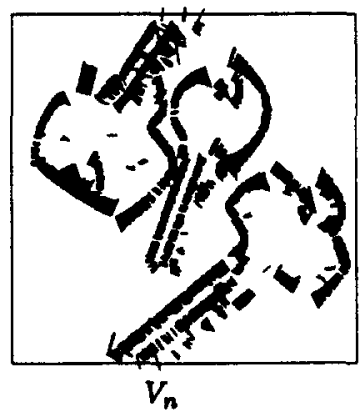

(b)

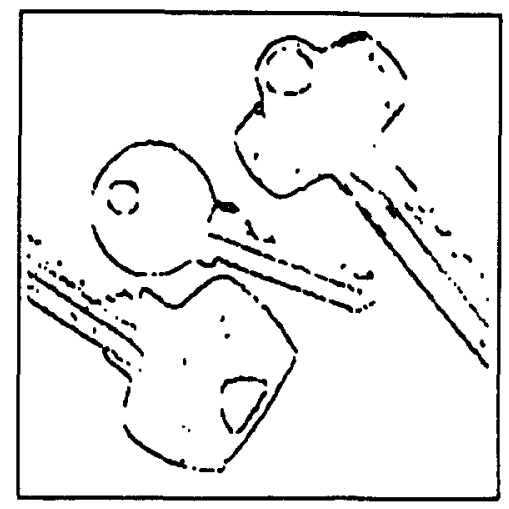

(c)

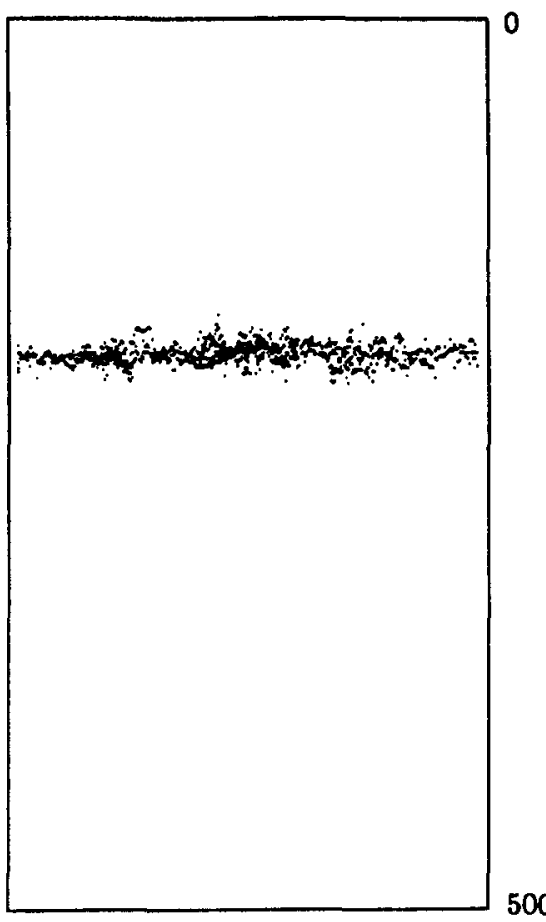

(d)

Fig. 10. Keys.

image points is two-dimensional; while for static stereo, the objects are practically stationary, the motion of the image points is one-dimensional. So the estimation of relative normal flow field from static stereo images is more accurate.

3.3.2. Keys. In the previous experiments, we dealt with synthetic images, which are perfectly designed and noise-free. Practically, such images can never be obtained. To see if an algorithm really works, we have to try it on real images. In this and the next experiments, we apply our algorithm to laboratory images. The images are taken by laterally spaced cameras.

In this experiment, the objects in the scene are three keys laying on a table. The keys are supposed to have the same depth values. The cameras are carefully positioned such that a point on the key plane has an image disparity of 2 pixels. If the distances between the cameras are set to be 400 units, the keys should be 200 units from the imaging plane.

Figure 10 shows the figures associated with this 


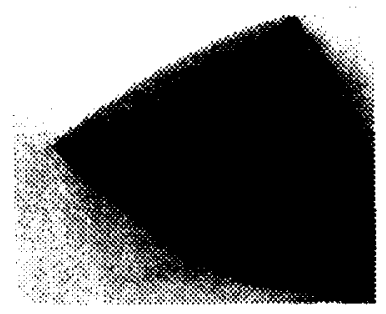

12

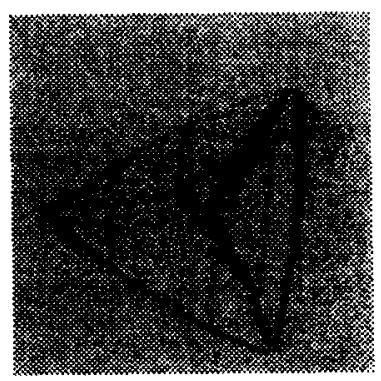

dp

(a)

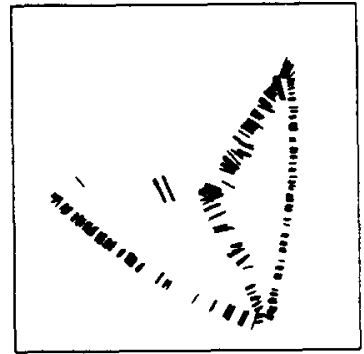

$V_{n}$

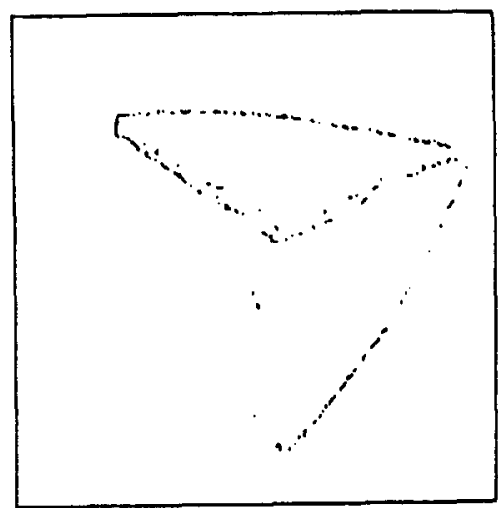

(c)

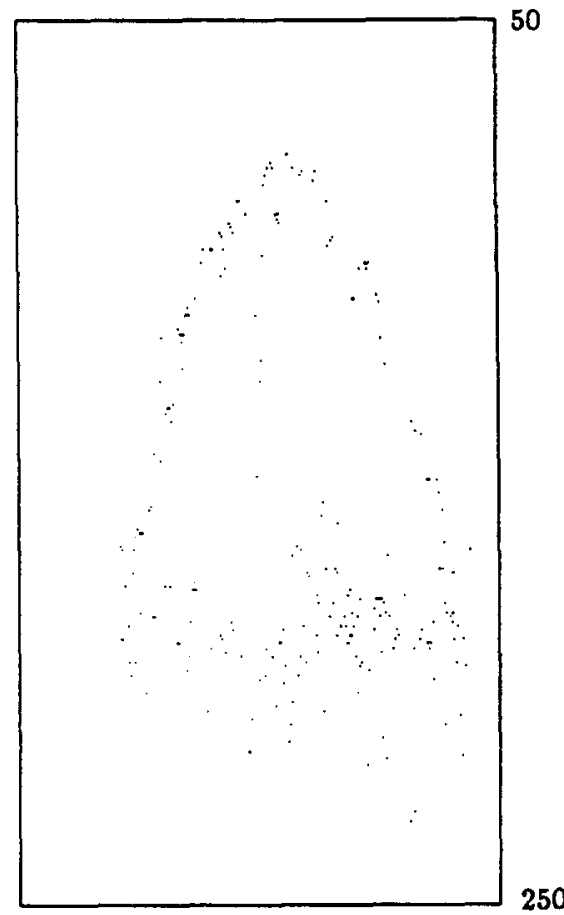

(d)

Fig. 11. Real pyramid.

experiment. The figures in Fig. 10 have similar explanations as those in Fig. 9, except that I2 in Fig. 10 (a) shows the original image, instead of having the background intensity values changed. The maximum relative error of the relative normal flow field is $5 \%$.

3.3.3. Pyramid. In the experiment. the imaging system setup is the same as that for the keys. The object is a four-side paper pyramid, the object points have different depth values from the bottom of the pyramid to the top. The algorithm should be able to recover the variant depth values. The base of the pyramid is set at a depth of 200 units.

Figure 11 shows the figures associated with this experiment. The figures in Fig. 11 have similar explanations as those in Fig. 10. As can be seen from Fig. 11(d), the computation result successfully shows the shape of the pyramid. For most of the strong edge points, the error is within $10 \%$.

As can be seen from the intensity image $I_{2}$, the intensity contrast is not high, the intensity gradient values are therefore small. This partially contributes 
to the relatively larger error in relative normal flow estimation.

\section{CONCLUSION}

Most of the depth from image flow algorithms fails to give satisfactory results either due to the fact that they are based on solving non-linear equations, which require good initial guesses, or due to the fact that they rely on some assumptions like planar surfaces, which limit the applications of the algorithms. Waxman and Sinha developed an algorithm that recovered depth from relative image flows to overcome the aforementioned difficulties.

In this paper, we extended Waxman and Sinha's work, and developed an algorithm to recover depth from relative normal flows. It is shown that normal flow provides information as good as optical flow for depth recovery purposes. Since normal flows can be estimated directly from image intensities, while the computation of optical flow is difficult and erroneous, our algorithm points out a new direction in passive ranging.

We noticed that if the normal flows were assumed given, depth computation would become simple and straightforward, and experiments would be of little interest. Hence, we tried our algorithm on intensity images. We have implemented a normal flow estimation algorithm. The normal flows are first estimated from the images, and the depth is then computed from the normal flow based on our algorithm. The results of the experiments are encouraging.

\section{REFERENCES}

1. H.-H. Nagel and B. Neuman, On 3D reconstruction from two perspective views, Proc. IJCAI, pp. 661-663 (1981).
2. J. W. Roach and J. K. Aggarwal, Determining the movement of objects from a sequence of images, IEEE PAMI-2, 554-562 (1980).

3. S. Ullman, The Interpretation of Visual Motion. MIT Press, Cambridge, MA (1979).

4. G. Adiv, Determining three-dimensional motion and structure from optical flow generated by several moving objects, IEEE PAMI-7, 384-401 (1984).

5. B. J. Buxton, D. W. Murray, H. Buxton and N. S Williams, Structure-from-motion algorithms for computer vision on an SIMD architecture, Comp. Phvs. Comm. 37, 273-280 (1985).

6. J.-Q. Fang and T. S. Huang, Solving three-dimensiona small-rotation motion equations: uniqueness, algorithms, and numerical results, CVGIP 26, 183-206 (1984).

7. K. Kanatani, Structure and motion from optical flow under orthographic projection, CVGIP 35, 181-199 (1986).

8. S. Negahdaripour and B. K. P. Horn, Direct passive navigation, IEEE PAMI-9, 168-176 (1987).

9. K. Sugihara and N. Sugie, Recovery of rigid structure from orthographically projected optical fiow, CVGIP 27, 309-320 (1984)

10. R. Y. Tasi and T. S. Huang, Estimating 3D motion parameters of a rigid planar patch, IEEE Acoust. Speech Sig. Process. 29, 1147-1152 (1981).

11. R. Y. Tsai and T. S. Huang, Uniqueness and estimation of three-dimensional motion parameters of rigid objects with curved surfaces, IEEE PAMI-6, 13-27 (1984).

12. X. Zhuang and R. M. Haralick. Rigid body motion and optic flow image, Proc. IEEE Conf. Artificial Intell. Alication, pp. 366-375 (1984)

13. A. M. Waxman and S. Sinha, Dynamic stereo: passive ranging to moving objects from relative image flows, IEEE PAMI-4, 406-412

14. T. D. Williams, Depth from camera motion in a real world scene, IEEE PAMI-2, 511-516 (1980).

15. M. Subbarao and A. M. Waxman, On the uniqueness of image flow solutions for planar surfaces in motion. Proc. IEEE Workshop on Computer Vision, pp. 129 $140(1985)$.

16. B. K. P. Horn and B. G. Schunck, Determining optical flow, Artif. Intell. 17, 185-203 (1981).

About the Author-FAN JIANG received the B.S. degree from the University of Science and Technology of China in 1983, and the M.S.E. degree from the University of Michigan in 1984. Currently he is a research assistant in the Electrical Engineering and Computer Science Department of the University of Michigan, and is working toward the Ph.D. degree in computer science. His research interests are in computer vision, motion detection and image sequence analysis.

\begin{abstract}
About the Author-TERRY E. WEYMOUTH received the B.S. and the M.S. degrees from the University of Nebraska in 1973, and 1976 respectively. He was awarded the Ph.D. degree in Computer Science in 1986 for his work on VISIONS, a system for interpreting natural scenes, developed at the University of Massachusetts at Amherst. From 1976 to 1979 he was employed by the Bell Laboratories, Naperville, Illinois. Since fall of 1985 he has been on the faculty of Electrical Engineering and Computer Science at the University of Michigan where he does research on computer vision, and artificial intelligence, and teaches courses in computer vision and graphics. Dr Weymouth is a member of IEEE, American Association for Artificial Intelligence, Association for Computing Machinery, and Upsilon Pi Epsilon (Computer Science Honors Society).
\end{abstract}

